\title{
Marchés avec coûts d'information sur la qualité des biens: Une application aux produits écolabellisés
}

\author{
Douadia BOUGHERARA ${ }^{1}$ \\ INRA ESR (Rennes) \\ Virginie PIGUET \\ INRA CESAER (Dijon)
}

\section{Résumé}

La théorie des défaillances de marché identifie les situations pour lesquelles la "main invisible" ne mène pas à une allocation efficace. Les marchés sont notamment dits défaillants en asymétrie d'information. Ce cadre est souvent évoqué pour analyser le rôle des labels dans les marchés pour des biens de croyance. Les labels sont alors des mécanismes permettant de rétablir la symétrie d'information entre vendeur et acheteur et de mener à des transactions plus efficaces. Nous montrons que les labels n'augmentent pas nécessairement l'efficacité des marchés car ils sont susceptibles d'introduire des coûts supplémentaires, sources d'inefficacité. Bien que notre étude soit généralisable, nous l'appliquons aux labels environnementaux. Une définition des attributs des biens en termes de coûts d'information sur la qualité enrichit l'analyse économique de la labellisation. Nous mettons en évidence, à travers une analyse expérimentale, l'inefficacité des marchés avec coûts d'information sur la qualité.

Mots clés: Coûts d'information; Biens de croyance; Economie expérimentale

Codes JEL: D43; D83; L15; Q50;

\footnotetext{
${ }^{1}$ Auteur chargée de la correspondance: INRA ESR - 4 allée Bobierre CS 61103, 35011 Rennes cedex Tél. (33) 02234856 03, Fax.(33) 02234853 80, douadia.bougherara@rennes.inra.fr
} 


\section{Marchés avec coûts d'information sur la qualité des biens: Une application aux produits écolabellisés}

\section{Introduction}

La diversité des programmes d'étiquetage ou de labellisation des produits apparaît notamment dans leur contenu puisqu'ils portent sur une grande variété d'attributs des biens, allant d'attributs intrinsèques à des attributs extrinsèques tels que l'utilisation d'hormones ou le recours au travail des enfants. L'étiquetage a un rôle particulièrement important lorsqu'il s'agit d'attributs de croyance des biens, attributs qui sont difficilement vérifiables avant et après l'achat. Par exemple, à la fin des années 70, des programmes d'écolabellisation sont apparus dans le cadre des instruments de politiques d'environnement. Ils sont en expansion et visent à constituer, dans leur principe, un moyen décentralisé de production de biens publics.

L'étiquetage a souvent été analysé dans le cadre de la théorie des défaillances de marché, théorie qui identifie les situations pour lesquelles la "main invisible" des marchés est prise en défaut et ne peut mener à une allocation efficace. En ce qui concerne les attributs environnementaux, l'information étant asymétrique entre vendeur et acheteur, des phénomènes de sélection adverse apparaissent : les produits de haute qualité sont éliminés du marché (Akerlof, 1970). L'étiquetage des produits, en rétablissant la symétrie d'information entre vendeur et acheteur, permet de répondre à la demande de biens de qualité supérieure et donc de mener à des transactions plus efficaces (Teisl, Roe et Hicks, 2002; McCluskey, 2000; Caswell et Mojduzska, 1996).

Notre article apporte une autre perspective à l'analyse des politiques, notamment environnementales, d'étiquetage des biens de croyance. Si le cadre de l'asymétrie d'information est déjà riche, nous suggérons que le rétablissement de la symétrie d'information n'est pas une solution suffisante aux problèmes des biens de croyance. En effet, même avec un écolabel, les coûts d'information subis par les acteurs lors de la transaction peuvent rester relativement élevés. Il peut en résulter une dissipation des consentements à payer des consommateurs dans une recherche et un traitement coûteux de l'information fournie. Le consentement à payer, levier fondamental et déjà fragile des politiques d'éco- 
étiquetage, est mis à mal. Nous utilisons l'économie expérimentale qui a l'avantage de permettre un contrôle des variables considérées. En effet, il est dans la réalité difficile de mesurer le coût réel supporté par les acheteur pour acquérir l'information sur la qualité des produits. En laboratoire, ce coût est fixé par l'expérimentateur à différents niveaux afin d'observer la réaction du marché. Alors que la littérature expérimentale a très tôt considéré le comportement des marchés en asymétrie d'information, elle n'a jusqu'à présent pas considéré les marchés avec coûts d'information sur la qualité des produits. Nous menons ainsi une analyse de l'efficacité des marchés non plus en présence d'asymétrie totale d'information mais en présence de coûts variables d'information sur la qualité.

Notre article est organisé comme suit. Dans la partie II, nous réalisons une revue de la littérature théorique et expérimentale sur les asymétries d'information et les marchés avec coûts d'information sur la qualité. La partie III analyse les sources de coûts d'information sur la qualité pour les produits écolabellisés. Ensuite, nous présentons le protocole expérimental utilisé (partie IV). Les modèles et hypothèses de travail font l'objet de la partie V. Enfin, nous présentons et discutons les principaux résultats (partie VI) et la partie VII conclut.

\section{Revue de la littérature}

Nous réalisons une revue sommaire de la littérature théorique et expérimentale dans deux domaines: les marchés imparfaits et les coûts d'information sur la qualité.

\section{A. Littérature sur les marchés imparfaits}

\section{a. Littérature théorique}

L'économie de l'information classe les biens en trois catégories: les biens de recherche, d'expérience et de croyance.

Dans la théorie de recherche d'information de Stigler (1961), le consommateur poursuit sa recherche d'information tant que le coût marginal de cette information n'excède pas son bénéfice marginal espéré. Stigler ne traitait pas de la recherche d'information sur la qualité des produits mais de la recherche du prix le plus faible d'un produit homogène dans différents points de vente. Pour Nelson (1970), le bien étudié par Stigler est un bien de recherche, bien 
pour lequel les acheteurs peuvent inspecter les diverses alternatives avant l'achat. Il introduit une nouvelle catégorie, les biens d'expérience, pour lesquels il vaut mieux acquérir l'information sur la qualité par l'achat du bien que par la collecte d'information avant l'achat. Ainsi, le coût de recherche de l'information avant l'achat est supérieur au prix du bien. Il est préférable d'acheter de l'information à travers "l'expérience" du produit, c'est-à-dire l'achat et la consommation du produit, tant que le coût marginal de l'information acquise de cette manière est inférieur à son bénéfice marginal. Enfin, dans leur article séminal, Darby et Karni (1973) ajoutent la catégorie des biens de croyance, dont la qualité est coûteuse à déterminer même après l'achat. D'autres auteurs reprennent cette classification en termes de coûts d'acquisition de l'information sur la qualité par les acheteurs (Ford et al., 1988; Krouse, 1990, p. 510; Andersen et Philipsen, 1998; Cho et Hooker, 2002).

La revue de la littérature théorique suggère donc une classification des biens en fonction du coût d'acquisition de l'information sur la qualité par les acheteurs plutôt que sur le moment où le consommateur acquiert l'information sur la qualité (avant, après l'achat ou jamais). Ainsi, les biens sont distribués selon un continuum, du bien de recherche parfait, pour lequel les coûts d'acquisition de l'information sont nuls au bien de croyance parfait, pour lequel ils sont prohibitifs.

\section{b. Littérature expérimentale}

Les expérimentalistes se sont très tôt intéressés aux marchés avec asymétrie d'information ${ }^{2}$. Les questions traitées sont diverses.

Plott et Wilde (1982) étudient l'efficacité ${ }^{3}$ des marchés où l'information sur les besoins des consommateurs est détenue par les vendeurs, en concurrence. Dans leur protocole, les acheteurs peuvent, sans coûts, hypothèse forte, consulter différents vendeurs pour obtenir un diagnostic de leurs besoins avant d'acheter un service. La concurrence entre les vendeurs diminue l'incitation des vendeurs à frauder (par la sur-prescription de services) et augmente l'efficacité du marché.

\footnotetext{
${ }^{2}$ Etant donné notre problématique, nous nous focalisons sur la littérature expérimentale traitant de la sélection adverse. Pour l'aléa moral, voir Keser et Willinger (2002; 2000), DeJong et al. (1985).

${ }^{3}$ On entend ici par efficacité la capacité du marché à permettre l'échange de biens ou de services de qualité supérieure.
} 
Miller et Plott (1985) s'intéressent à la capacité du signalement à pallier la sélection adverse, en menant à un équilibre séparateur. Le type du vendeur (vendeur de biens de qualité faible ou élevée) est fixé de manière exogène. Les vendeurs ont la possibilité d'ajouter des unités de qualité q coûteuses à leurs biens, q coûtant plus cher aux vendeurs de type faible qu'aux vendeurs de type élevé ; q joue le rôle de signal de qualité. Leurs principaux résultats sont que l'existence d'un équilibre séparateur dépend de la différence de coûts des unités q entre vendeurs de type faible et vendeurs de type élevé (prédiction de la théorie du signal).

Lynch et al. (1991; 1986) mettent en évidence la sélection adverse dans les marchés avec asymétrie d'information. La révélation publique de la qualité des biens après l'achat est plus efficace que la révélation privée. Les stratégies de réputation individuelle des vendeurs sont difficiles à établir car les acheteurs sont influencés par la réputation totale du marché. Les garanties rendues exécutoires réduisent la sélection adverse, ce qui n'est pas le cas des garanties auto-déclarées. Un résultat intéressant pour notre discussion est que les auteurs n'ont trouvé aucune différence significative sur l'efficacité de marché entre le traitement où la qualité des biens était révélée après chaque achat (bien d'expérience) et celui où la qualité n'était révélée qu'après huit périodes.

Enfin, Cason et Gangadharan (2002) analysent la réputation, l'auto-déclaration et la certification comme moyens d'augmenter l'efficacité de marchés avec sélection adverse. La réputation et l'auto-déclaration augmentent moyennement l'efficacité du marché. La certification est le seul traitement où la plupart des unités échangées sont de qualité supérieure.

On remarque que la plupart de ces articles traitent de biens d'expérience. Deux articles analysent le marché des biens de croyance : Plott et Wilde (1982) traitent uniquement du problème de diagnostic du bien d'expert et Lynch et al. (1991) du problème de vérification.

\section{B. Littérature sur les marchés avec coûts d'information sur la qualité}

De manière surprenante, peu d'articles traitent des marchés avec coûts d'information sur la qualité en comparaison de ceux qui s'intéressent à la recherche coûteuse d'information sur les prix. Néanmoins, deux travaux théoriques sont à noter. 
Kihlstrom (1974) propose une théorie générale de la demande d'information sur la qualité des produits. Il prête à cette demande d'information deux caractéristiques. Premièrement, c'est une demande dérivée. Elle n'existe que parce qu'elle permet aux consommateurs d'effectuer de meilleurs choix dans l'achat d'autres biens. Deuxièmement, la demande d'information n'apparaît que lorsque la qualité des produits est incertaine.

A partir du modèle de Grossman et Stiglitz (1980), Bester et Ritzberger (2001) développent un modèle de marché avec coûts d'information sur la qualité (jeu dynamique à information incomplète). Les vendeurs sont de 2 types (offreurs de biens de qualité inférieure $q_{L}$ et offreurs de biens de qualité élevée $q_{H}$ ). Les acheteurs savent que les vendeurs fournissent la qualité $q_{H}$ avec la probabilité $l \in[0,1]$ et la qualité $q_{L}$ avec la probabilité $(1-l)$. Après avoir observé le prix du bien, chaque acheteur peut tester la qualité du bien en payant un coût fixe $k>0$, révélant parfaitement la qualité. Les auteurs montrent que l'équilibre de ce type de jeu dépend des croyances des agents sur les comportements des autres agents. Sous certaines conditions, plus le coût d'information est faible, plus les prix révèlent l'information et donc moins les acheteurs auront tendance à investir dans l'information.

Dans la littérature expérimentale, on note l'étude de Brannon et Gorman (2002) qui étudient notamment le rôle de la connaissance par les acheteurs de la dispersion des prix sur les performances de marchés avec coûts d'information sur les prix. Cependant, la littérature expérimentale n'a à notre connaissance pas traité des marchés avec coûts d'information sur la qualité des biens.

\section{Les produits éco-labellisés: des coûts élevés d'information sur la qualité}

Les produits éco-labellisés font souvent partie de la catégorie des biens de croyance. Ainsi, les coûts d'information sur la qualité environnementale des biens sont rarement faibles ${ }^{4}$. Nous identifions trois sources de coûts d'information sur la qualité environnementale (Karl et Orwat, 1999).

\footnotetext{
${ }^{4}$ Certains attributs environnementaux peuvent cependant présenter de faibles coûts d'information. C'est le cas si un écolabel indique l'utilisation moindre d'emballage. D'un simple coup d'œil, le consommateur détermine si le dentifrice est vendu avec ou sans emballage cartonné.
} 


\section{A. Coûts de définition de la qualité environnementale}

La définition de l'écoproduit n'est pas absolue mais relative. Elle est endogène au processus de qualification des produits (Nadaï, 1998). La difficulté provient du fait que le consommateur est dans l'incapacité de déterminer ce qu'est un produit respectueux de l'environnement, du fait par exemple de son manque de capacité à juger de la qualité du bien. Le problème n'est pas de vérifier si une pratique a été mise en œuvre (observabilité parfaite des actions du vendeur) mais de déterminer la pertinence de la pratique pour le respect de l'environnement (Plott et Wilde, 1982). Le matériau utilisé pour ce produit est-il ou non préférable pour l'environnement?

A défaut de pouvoir définir de manière absolue "le" produit respectueux de l'environnement, il s'agit d'établir des règles du jeu (par exemple, participation de l'ensemble des parties dans la définition) permettant d'assurer un certain niveau de loyauté des moyens mis en ouvre à défaut de garantir la qualité du résultat.

\section{B. Cô̂ts de vérification de la qualité environnementale}

Il s'agit de la vérification ou de la mesure de la conformité avec la définition préalable. Cette phase est étroitement dépendante de la précédente, qui définit parfois de manière plus ou moins précise les modalités de la vérification. Cette seconde source de coûts est due à l'incapacité de l'acheteur à vérifier ou à observer la qualité du produit. Les caractéristiques environnementales se réfèrent souvent au processus de production.

Les acheteurs sont alors contraints de s'appuyer sur des indicateurs de qualité tels que la présence de l'écolabel, la réputation du vendeur ou la certification par une tierce partie.

\section{Cô̂t de signalement de la qualité environnementale}

Enfin, même si les consommateurs identifient leurs besoins (définition) et ont confiance dans l'écolabel (vérification), il peut exister une autre source de coûts. Nous souhaitons ici distinguer l'asymétrie d'information de la surcharge informationnelle, même si la distinction n'est pas toujours très nette. L'asymétrie d'information correspond à une répartition inégale d'information entre deux agents. Cette distribution inégale permet à l'agent le mieux informé, 
par exemple le vendeur, de tirer profit de son information privée. La surcharge d'informations provient des capacités cognitives limitées des individus. Même dans un monde caractérisé par la symétrie d'information, les agents, ici, les consommateurs, sont submergés de flots croissants d'information. C'est l'attention du consommateur et non l'information qui devient une ressource rare (Davenport et Beck, 2001).

La prolifération des labels sur les produits et le peu de temps alloué aux achats par les consommateurs fait de la lecture et du traitement de l'information par les consommateurs une activité coûteuse, en efforts et en temps. L'efficacité d'un écolabel est ainsi, en grande partie, fonction de la clarté du message qu'il véhicule. Si les consommateurs ne peuvent traiter ce message alors leurs coûts d'information vont augmenter (Loader et Hobbs, 1999), alors que le dispositif d'écolabellisation vise justement à économiser sur les coûts.

Ainsi, en plus de la réaction du marché à une asymétrie totale d'information, il est pertinent d'étudier son comportement en présence de coûts variables d'information. Nous présentons dans les sections suivantes une étude expérimentale de ces marchés.

\section{Protocole expérimental}

Nous présentons le cadre expérimental utilisé, similaire à celui de Cason et Gangadharan $(2002)^{5}$ et de Lynch et al. (1991) ainsi que les 3 traitements.

\section{A. Cadre expérimental}

Les participants de l'expérience sont des étudiants en sciences (99) de l'Université de Bourgogne (Dijon), de l'Etablissement National d'Enseignement Supérieur Agronomique de Dijon et de l'Université de Rennes, sélectionnés par e-mail ou par annonce en amphithéâtre. Chaque session compte 11 participants : 5 vendeurs et 6 acheteurs. Le rôle de chaque participant est déterminé de manière aléatoire. Les expériences ont eu lieu à l'ENESAD (Dijon) et au LABEX (Rennes) dans une salle informatique où les participants étaient isolés les uns des autres par des cloisons. Le logiciel REGATE (CNRS, Ecully) a permis la programmation et le déroulement de l'expérience. L'utilisation de cloisons ainsi que d'un

\footnotetext{
${ }^{5}$ Le protocole a été rédigé lors d'un séjour à Purdue University. Nous sommes reconnaissant à Tim Cason pour son accueil et sa précieuse aide.
} 
logiciel permet de contrôler l'environnement de l'expérience notamment la possibilité d'une communication qu'elle soit verbale ou non. Les instructions sont lues à l'ensemble des participants en début d'expérience (voir annexe pour un exemple d'instructions). Les participants découvraient ensuite leur rôle et recevaient leur feuille d'information privée incluant les valeurs de reprise pour les acheteurs et les coûts de production pour les vendeurs. Nous avons réalisé plusieurs séances pilotes pour affiner le protocole, les instructions et le fonctionnement du programme informatique. Les exemples utilisés dans les instructions présentaient des valeurs délibérément élevées afin d'éviter qu'ils influencent le comportement dans l'expérience.

On considère un marché avec vendeurs et acheteurs avec préférences induites ${ }^{6}$. Nous avons réalisé 9 sessions, chacune durant 3 à 4 heures. Toutes les sessions, comportaient 32 périodes d'échange, exceptée la première (24). Les participants ont réalisé leurs échanges en points qui étaient convertis en euros au cours et à la fin de l'expérience, selon un taux de conversion défini préalablement. Ce taux de conversion était 1 point $=0,004 €$ pour les vendeurs et 1 point $=0,013 €$ pour les acheteurs. Les gains moyens par participant étaient de $30 €$. Ceux-ci étaient rémunérés à la fin de l'expérience.

A chaque période de marché, les vendeurs peuvent vendre au maximum deux unités de type I (Inférieur) ou deux unités de type S (Supérieur). Le fait que les unités S soient plus coûteuses à produire que les unités I est information publique mais seuls les vendeurs connaissent les valeurs des coûts de production. Chaque unité S coûte 120 points aux vendeurs et chaque unité I, 20 points. Les valeurs de reprise des acheteurs pour des unités S sont supérieures aux valeurs de reprise pour des unités I. C'est une information publique, mais seuls les acheteurs en connaissent les valeurs exactes. La valeur marginale des unité S est toujours supérieure à la valeur marginale des unités I. La valeur de la première unité S est de 330 points, celle de la seconde unité est de 300 points et celle de la troisième unité est de 270 points. Pour les unités I, les valeurs sont respectivement 180, 165 et 150. Par conséquent, les acheteurs préfèrent acheter des unités $\mathrm{S}$ à moins que le prix soit supérieur de 120 à 150 points au prix des unités I.

\footnotetext{
${ }^{6}$ On distingue généralement deux types d'expériences selon l'objectif poursuivi. Dans les expériences d'évaluation, on fixe les règles du marché, et on observe les préférences des agents (homegrown values). Dans les expériences avec préférences induites, on fixe les préférences des agents et on compare différentes institutions de marché (induced values).
} 
Tous les acheteurs et les vendeurs ont des valeurs de reprise et des coûts de production identiques. La structure de l'offre et de la demande est indiquée sur la figure 1.

\section{[Insérer la figure 1]}

Théoriquement, à l'équilibre du marché parfaitement efficace, où seules des unités $\mathrm{S}$ sont échangées, le prix d'équilibre est de 300 avec 10 unités échangées. Cet équilibre correspond à un surplus total, pour l'ensemble des participants, de 1980 points $^{7}$. A l'équilibre de marché inefficace, où seules des unités I sont échangées, le prix d'équilibre est de 165 avec 10 unités échangées. Cet équilibre correspond à un surplus total de 1540 points.

A la manière de Cason et Gangadharan (2002), nous définissons l'efficacité de marché comme le rapport entre la somme des surplus réels de chacun des participants dans la session étudiée et la somme des surplus théoriquement que chacun des participants aurait gagné si seules des unités S avaient été échangées (1980 points). L'efficacité du marché à l'équilibre inefficient (seuls des unités I sont échangées) est alors 1540/1980=0,778. En plus des profits dus aux unités achetées, les acheteurs reçoivent un bonus de 50 points à chaque période et un gain de début d'expérience de 200 points, les acheteurs étant susceptibles, dans les premières périodes de l'expérience, d'essuyer des pertes. Le bonus et le gain initial permettent d'absorber les pertes et de maintenir l'incitation financière tout au long de l'expérience. Par ailleurs, nous donnons la possibilité aux acheteurs de ne pas acheter d'unités dans une période (option de non achat). Leur gain de non achat s'élève alors à 10 points.

\section{B. Traitements}

Le tableau 1 résume les 3 traitements (Ref, High et Low). Rappelons que l'objectif est de mesurer l'efficacité du marché et les comportements de recherche d'information lorsque les coûts d'information sont croissants et susceptibles d'absorber les consentements à payer, c'est-à-dire le surplus que les consommateurs peuvent retirer du bien. Dans le traitement Ref,

\footnotetext{
${ }^{7}$ A l'équilibre, 10 unités $\mathrm{S}$ sont échangées au prix 300:

- Pour les vendeurs: les 10 unités S rapportent un surplus de (300-120)=180 par unité soit 180x10=1800 points de surplus

- Pour les acheteurs: les 6 premières unités $\mathrm{S}$ correspondent à un prix de réserve de 330 alors que les 4 suivantes, à un prix de réserve de 300 . Les acheteurs gagnent ainsi un surplus de $(330-300)=30$ points par unité sur les 6 premières unités et un surplus de (300-300) $=0$ sur les 4 unités suivantes. Ainsi, le surplus total des acheteurs est: $30 \times 6+0 \times 4=180$.

Ainsi, le surplus total à l'équilibre est 1980 points, 1800 pour les vendeurs et 180 pour les acheteurs.
} 
nous mesurons l'efficacité du marché en présence d'asymétrie totale d'information. Dans ce traitement, il est impossible, pour les acheteurs, de vérifier la qualité des unités, le coût d'acquisition de l'information est pour ainsi dire "infini". Dans le traitement High, nous observons l'efficacité du marché en présence de coûts d'information élevés, 100 points, c'està-dire environ $66 \%$ du surplus le plus élevé du fait d'acheter une unité S plutôt qu'une unité $\mathrm{I}^{8}$. Dans le traitement Low, nous mesurons l'effet de la possibilité pour les acheteurs d'obtenir l'information à un faible coût de 15 points, c'est à dire $10 \%$ du surplus le plus élevé du fait d'acheter une unité S plutôt qu'une unité I.

\section{[Insérer le tableau 1]}

Nous avons conduit trois sessions pour chacun des traitements. Les vendeurs indiquent au début de chaque période le nombre d'unités qu'ils souhaitent proposer, le prix unitaire ainsi que le type des unités choisi. Le tableau de l'ensemble des offres (prix et nombre d'unités) est transmis aux acheteurs dans un ordre aléatoire pour cacher l'identité des vendeurs et contrôler les phénomènes de réputation. Les acheteurs sont alors choisis de manière aléatoire et séquentielle pour réaliser leurs achats (Holt, 1995, pp.361-365).

La session est divisée en 4 parties. L’information sur le type des unités est révélée après chaque période en partie 1, après 4 périodes en partie 2, après 8 périodes en partie 3 et après 16 périodes en partie 4. Quand tous les acheteurs ont eu l'occasion d'acheter ou que toutes les unités ont été vendues, les types des unités sont révélées après $1,4,8$ ou 16 périodes selon la partie. Cette manière de procéder permettait aux acheteurs de se familiariser avec l'expérience (apprentissage des règles du jeu, des modes de calcul des gains, etc.) surtout pendant les 4 premières périodes. Il semblait en effet difficile de mettre en œuvre le protocole directement, car alors les acheteurs n'auraient eu aucun retour sur leurs décisions.

\section{Modèle et hypothèses}

Nous nous intéressons d'une part, à l'efficacité du marché (propension à permettre l'échange d'unités de type $\mathrm{S}$ ) et à la probabilité pour les vendeurs de proposer des unités de type $\mathrm{S}$ et d'autre part, au comportement de demande d'information des acheteurs.

\footnotetext{
${ }^{8}$ La valeur de reprise d'une unité de type S (respectivement de type I) est de 330 (respectivement de 180) au maximum. Le consentement à payer maximum des acheteurs pour une unité de type $\mathrm{S}$ par rapport à une unité de type I est donc 330-180=150.
} 


\section{A. Efficacité du marché et probabilité de proposer des unités de type $S$}

Deux modèles sont susceptibles de s'appliquer selon le traitement considéré : un modèle d'information parfaite et un modèle de sélection adverse.

\section{a. Modèle d'information parfaite}

Dans ce modèle, les unités sont échangées au prix d'équilibre du marché. Les unités $\mathrm{S}$ sont échangées au prix de 300 et les unités I au prix de 165 (voir figure 1). Comme, les unités S génèrent un flux de profit supérieur à celui généré par les unités I, l'équilibre prédit est que seules des unités $\mathrm{S}$ seront échangées à leur prix d'équilibre 300 .

Les sessions du traitement Low seront susceptibles de se rapprocher de ce modèle d'information parfaite. "As the information cost becomes negligible, the equilibrium approaches the full information outcome and prices become perfectly informative" (Bester et Ritzberger, 2001, p.1360).

\section{b. Modèle de sélection adverse}

Selon ce modèle développé par Akerlof (1970), les biens de haute qualité sont éliminés du marché car les acheteurs, incapables de vérifier la qualité des biens, n'accepteront pas de payer un prix supérieur au prix de la qualité moyenne sur le marché. Les vendeurs de haute qualité ne seront donc pas incités à mettre des biens de haute qualité sur le marché. Seuls des biens de qualité basse seront échangés. Seules des unités I seront échangées au prix d'équilibre de 165. Les marchés dans les sessions des traitements Ref et High sont susceptibles de se rapprocher de cet équilibre.

\section{B. Comportement de demande d'information sur la qualité des biens}

\section{a. Effet du coût de l'information}

Concernant la demande d'information, la loi de la demande prédit que plus l'information sera coûteuse moins les participants investiront dans la recherche d'information. On s'attend à ce 
que le nombre de demandes d'information dans le traitement High soit inférieur au nombre de demandes d'information dans le traitement Low.

\section{b. Influence du prix des biens sur la demande d'information}

L'influence du prix des unités sur la demande d'information sur la qualité des biens est moins évidente. La relation de la demande d'information au prix n'est pas linéaire.

En effet, il semble logique de considérer qu'il existe:

- un prix au-dessous duquel les acheteurs n'achèteront pas d'information sur la qualité du fait qu'ils supposent que l'unité est de qualité inférieure

- et un prix au-dessus duquel les acheteurs n'achèteront pas d'information étant donné qu'ils n'achèteront pas l'unité même si elle est de type $\mathrm{S}$.

En se basant sur le modèle de Bester et Ritzberger (2001), soient $u$ le profit en cas de non achat, $p$ le prix de l'unité, $\mu(p)$ la croyance de l'acheteur selon laquelle l'unité est de type $\mathrm{S}$ étant donnée son prix $p, V$ la valeur de reprise de l'unité qui vaut $V_{I}$ si l'unité est de qualité inférieure, et $V_{S}$ si l'unité est de qualité supérieure

Alors, les profits espérés de l'acheteur en n'achetant pas d'information sont:

$\operatorname{Max} \quad\left[\mu(p) \cdot V_{S}+(1-\mu(p)) \cdot V_{I}-p, u\right]$

L'acheteur, une fois informé, achète le bien si $V-p \geq u$. Le profit espéré du fait d'acheter de l'information est alors:

$\mu(p) \cdot \operatorname{Max}\left[V_{S}-p, u\right]+(1-\mu(p)) \cdot \operatorname{Max}\left[V_{I}-p, u\right]-k$ avec $k$, le coût de l'information.

L'objectif est de déterminer le comportement d'achat d'information sur les unités par les acheteurs. Deux cas se présentent alors:

- De manière évidente, si $u>V_{S}-p$, les acheteurs n'achèteront pas l'unité et donc n'investiront pas dans une recherche d'information coûteuse. En effet, si l'unité est de type S, elle apportera un gain inférieur au gain de non achat. Comme $u=10$, les acheteurs n'achètent 
pas d'unité et donc pas d'information si $p>V_{S}-u$ soit 320 pour la première unité, 290 pour la deuxième unité et 260 pour la troisième unité 9 .

- De la même manière, si $u<V_{I}-p$, les acheteurs achèteront l'unité sans chercher à acheter de l'information sur le type des unités. L'achat de l'unité apporte de toute manière un gain supérieur au gain de non achat. De même, les acheteurs achètent l'unité sans chercher à acheter de l'information sur l'unité si $p<V_{I}-u$ soit 170 pour la première unité, 155 pour la deuxième unité et 140 pour la troisième unité.

Nous formulons donc l'hypothèse suivante:

La demande d'information sera nulle lorsque le prix de l'unité appartient:

- à l'ensemble ] $-\infty, 170] \cup[320,+\infty[$ si l'acheteur n'a pas encore acheté d'unités dans la période

- à l'ensemble ] - $\infty, 155] \cup[290,+\infty[$ si l'acheteur a déjà acheté 1 unité dans la période

- à l'ensemble $]-\infty, 140] \cup[260,+\infty[$ si l'acheteur a déjà acheté 2 unités dans la période

Les conclusions ne sont pas aussi claires lorsque $V_{I}-u<p<V_{S}-u$. Dans ce cas, l'acheteur investit dans l'information si le profit espéré avec achat d'information est supérieur au profit espéré sans achat d'information soit:

$$
\begin{aligned}
\mu(p) \cdot \operatorname{Max}\left[V_{S}-\right. & p, u]+(1-\mu(p)) \cdot \operatorname{Max}\left[V_{I}-p, u\right]-k \\
& \geq \operatorname{Max}\left[\mu(p) \cdot V_{S}+(1-\mu(p)) \cdot V_{I}-p, u\right]
\end{aligned}
$$

Comme $V_{I}-u<p<V_{S}-u$, on sait que $V_{S}-p>u$ et que $u>V_{I}-p$. Alors,

$$
\begin{aligned}
& \mu(p) \cdot\left(V_{S}-p\right)+(1-\mu(p)) \cdot u-k \\
& \geq \operatorname{Max}\left[\mu(p) \cdot V_{S}+(1-\mu(p)) \cdot V_{I}-p, u\right]
\end{aligned}
$$

Alors 2 cas se présentent:

\footnotetext{
${ }^{9}$ Rappelons que selon l'ordre d'achat de l'unité dans la période, $V_{S}-p=330-\mathrm{p}$ ou 300-p ou 270-p et $V_{I}-p=180-\mathrm{p}$ ou $165-\mathrm{p}$ ou $150-\mathrm{p}$.
} 
- Soit $\operatorname{Max}\left[\mu(p) \cdot V_{S}+(1-\mu(p)) \cdot V_{I}-p, u\right]=u$

Alors, $\mu(p) .\left(V_{S}-p\right)+(1-\mu(p)) . u-k \geq u$ soit $p \leq V_{S}-u-\frac{k}{\mu(p)}$

- Soit $\operatorname{Max}\left[\mu(p) \cdot V_{S}+(1-\mu(p)) \cdot V_{I}-p, u\right]=\mu(p) \cdot V_{S}+(1-\mu(p)) \cdot V_{I}-p$

Alors, $\mu(p) \cdot\left(V_{S}-p\right)+(1-\mu(p)) \cdot u-k \geq \mu(p) \cdot V_{S}+(1-\mu(p)) \cdot V_{I}-p$

soit $V_{I}-u+\frac{k}{1-\mu(p)} \leq p$

Après avoir observé $p$, il est optimal pour un acheteur d'investir dans une recherche coûteuse d'information si et seulement si:

$V_{I}-u+\frac{k}{1-\mu(p)} \leq p \leq V_{S}-u-\frac{k}{\mu(p)}$

Il est difficile de tester cette hypothèse à l'aide des données dont nous disposons puisque nous ne connaissons pas $\mu(p)$ pour notre échantillon. Cependant, on peut raisonner à travers le recoupement des intervalles sur lesquels l'achat d'information est susceptible d'être une stratégie optimale. Quatre seuils apparaissent dans notre discussion: 140, 170, 260 et 320.

Ainsi, si le prix de l'unité est inférieur à 170, aucune information ne sera achetée. De même, si le prix est supérieur à 320 . Si le prix est compris entre 170 et 260 , la probabilité de demander de l'information sera forte car quelque soit l'ordre d'achat des unités dans la période, il y aura demande d'information. Si le prix est compris entre 260 et 320, la probabilité de demander de l'information sera élevée mais moins forte que dans l'intervalle [170,260] car seules les $1^{\text {ères }}$ et $2^{\text {èmes }}$ unités sont concernées. Enfin, si le prix est compris entre 140 et 170 , la probabilité de demander de l'information sera élevée mais moins forte que dans l'intervalle [260,320] car seules les $2^{\text {èmes }}$ et $3^{\text {èmes }}$ unités sont concernées. Or, par définition, les $1^{\text {ères }}$ unités ont la fréquence d'interrogation sur l'opportunité d'achat la plus forte.

La section suivante présente les résultats sur l'efficacité des marchés expérimentaux mis en œuvre, la probabilité de proposer des unités de type S pour les vendeurs et la probabilité pour les acheteurs de demander de l'information sur les unités proposées à la vente. 


\section{Résultats}

Nous réalisons une analyse statistique descriptive puis économétrique de nos données. Les traitements considèrerons les périodes 5 à 32 de chaque session étant donné que les quatre premières périodes constituaient une phase d'apprentissage des règles du jeu par les participants.

\section{A. Analyse descriptive}

Le tableau 2 présente le nombre d'unités $\mathrm{S}$ et I proposées et l'efficacité du marché par traitement. Les périodes 5 à 32 sont d'abord considérées (rappelons que les 4 premières périodes sont exclues du traitement étant donné qu'elles constituent une période d'apprentissage) puis les 10 dernières périodes (23-32) puis les 5 dernières (28-32). Rappelons qu'étant donné que la session A ne comprenait que 24 périodes, celle-ci n'apparaît pas dans le tableau.

\section{[Insérer le tableau 2]}

On constate sur ces données que le nombre d'unité I (S) croît (décroît) avec le coût d'information. Les données du tableau 2 présentent le traitement High sans la session F. En effet, cette session est atypique. Le nombre d'unités $\mathrm{S}$ échangées est particulièrement élevé. Nous réalisons un test de rangs signés de Wilcoxon afin de comparer les sessions au sein du même traitement. Dans les traitements Ref et Low, les différences entre les 3 sessions sont non significatives ou significatives à $10 \%$. Cependant, dans le traitement High, la session F se distingue de la session $\mathrm{D}$ avec une significativité de 5\% et de la session E avec une significativité de $1 \%$. Une des explications possibles est que les étudiants appartenaient à la même promotion. Il est donc probable que le marché ait été coopératif et non plus anonyme. Etant donné son caractère "aberrant", nous excluons la session F du traitement économétrique.

\section{B. Analyse économétrique}

Nous examinons à présent les trois variables dépendantes suivantes: l'efficacité du marché, la probabilité de proposer des unités de type $\mathrm{S}$ et enfin la demande d'information. 


\section{a. Efficacité du marché}

Nous estimons en premier lieu l'efficacité du marché et introduisons un effet aléatoire session.

Les données rencontrent un problème commun à beaucoup de résultats expérimentaux : les marchés sont sujets à un phénomène de convergence. En effet, les résultats se révèlent différents en début et en fin de session du fait du phénomène d'apprentissage. La régression doit en tenir compte. Noussair et al. (1995, pp.473-4) proposent de prendre en compte l'effet de convergence des marchés expérimentaux par l'introduction d'effets de début de session et de fin de session (encadré 1).

\section{[Insérer l'encadré 1]}

Notre expérience comprend trois traitements : Ref, High et Low. Conformément à la procédure de l'encadré 1 et à l'analyse similaire de Cason et Gangadharan (2002), nous créons deux variables indicatrices pour désigner le traitement : High=1 pour le traitement High et 0 sinon et Low $=1$ pour le traitement Low et 0 sinon. Ceci nous permet de créer les variables High_init, High_fin, Low_init et Low_fin, afin de capter les effets de début et de fin de session.

Les variables de la régression et leur signification sont présentées dans le tableau 3 (la variable RATIO est définie plus tard car elle ne concerne pas l'efficacité).

\section{[Insérer le tableau 3]}

La spécification du modèle est alors :

$$
y_{t}=\beta_{0}+\beta_{1} \times \text { Ref_init }+\beta_{2} \times \text { II_init }+\beta_{3} \times \text { II_fin }+\beta_{4} \times \text { III_init }+\beta_{5} \times \text { III_fin }+u
$$

Les interactions $1 / t$ captent l'effet "périodes initiales" et les interactions $(t-1) / t$ captent l'effet "dernières périodes" (tendances de long terme). 
Le traitement de référence est le traitement Ref pour lequel les agents sont en asymétrie totale d'information. L'estimation est présentée dans le tableau 4.

\section{[Insérer le tableau 4]}

L'effet aléatoire session n'est pas significatif. La comparaison des variables Ref_init, High_init et Low_init avec respectivement Ref_fin, High_fin et Low_fin montre l'existence de phénomènes de convergence. Les marchés ne se comportent pas de la même manière en début et fin de session. Cela reflète l'apprentissage des joueurs tout au long de la session.

Les 2 variables qui nous intéressent par rapport aux hypothèses formulées sont: High_fin et Low_fin.

L'estimation va dans le sens de nos hypothèses. Ainsi, l'efficacité du marché est significativement (1\%) plus élevée dans le traitement Low par rapport au traitement High et au traitement Ref. Par ailleurs, l'efficacité du marché dans le traitement High n'est pas significativement différente de l'efficacité du marché dans le traitement Ref. En ce qui concerne l'efficacité, il semble qu'il existe un seuil au-delà duquel les marchés avec coûts d'information se comportent comme les marchés en asymétrie totale d'information.

On peut se demander si l'effet du traitement sur l'efficacité du marché est différenciée selon le type d'agent. Nous réalisons 2 estimations supplémentaires. L'une où on ne prend en compte que les surplus des vendeurs dans le calcul de l'efficacité et l'autre où on ne prend en compte que le surplus des acheteurs. Les résultats sont présentés dans les tableaux 5 et 6 .

\section{[Insérer le tableau 5 et le tableau 6]}

Nous tirons deux remarques des résultats. Pour les vendeurs, on obtient globalement les même résultats que précédemment sauf que l'efficacité du marché dans le traitement High devient significativement supérieure (5\%) de l'efficacité du marché dans le traitement Ref. Pour les acheteurs, le traitement n'a pas d'effet sur l'efficacité. Ainsi, même une légère imperfection de l'information (15 points) mène à un marché inefficace. 


\section{b. Offre d'unités de type $S$}

Nous souhaitons étudier la probabilité de proposer de la qualité supérieure en fonction du coût de l'information. Un élément supplémentaire à prendre ici en compte est l'auto-corrélation temporelle des résidus : les décisions de la période $\mathrm{N}$ dépendent des décisions à la période $\mathrm{N}$ 1. A la manière de Cason et Gangadharan (2002), nous ajoutons un terme, RATIO $=\frac{E\left(\pi_{S}^{N}\right)}{E\left(\pi_{I}^{N}\right)}$ qui représente le rapport des profits espérés de vendre des unités $\mathrm{S}$ par rapport aux profits espérés de vendre des unités I (cf. tableau 3).

En prenant l'exemple des unités I, si un vendeur a offert de telles unités pendant les (N-1) périodes précédentes et offre des I dans la période courante $(\mathrm{N})$, il actualise ses croyances sur les profits espérés par la formule suivante :

$$
E\left(\pi_{I}^{N}\right)=\frac{(N-1) \times E\left(\pi_{I}^{N-1}\right)+\pi_{I}^{N}}{N}
$$

avec $E\left(\pi_{I}^{N}\right)$, les profits espérés de la vente d'unités I à la période $\mathrm{N}$ et $\pi_{I}^{N}$, le profit réel de la mise sur le marché d'unités I en période N.

Nous réalisons un Logit avec effets aléatoires sur les individus et définissons la variable TYPS=1 lorsque l'unité proposée par les vendeurs a une qualité supérieure et 0 lorsque sa qualité est inférieure. Le tableau 7 présente l'estimation.

\section{[Insérer le tableau 7]}

L'effet aléatoire est significatif à 1\%. Les variables High_init et Low_init ne seront pas commentées. Elles révèlent les mêmes phénomènes que précédemment. Seules les variables High_fin, Low_fin et RATIO seront considérées.

Le coefficient de la variable RATIO est toujours significatif et positif, ce qui va dans le sens de la théorie. Plus l'espérance de gain de proposer une unité S plutôt qu'une unité I est forte, plus les vendeurs ont tendance à proposer des unités de type $\mathrm{S}$. 
La probabilité de proposer des unité de type $\mathrm{S}$ est significativement supérieure mais légèrement (1\%) dans le traitement Low par rapport aux traitements High et Ref. Par ailleurs, les traitements High et Ref sont non significativement différents.

\section{c. Demande d'information sur la qualité}

Nous définissons la variable DEM=1 lorsque de l'information est demandée sur l'unité considérée et 0 sinon. Les traitements considérés ici sont les traitements où les agents avaient la possibilité de demander de l'information sur la qualité des unités, soit les traitements High et Low. La référence est le traitement Low. Les acheteurs ne pouvaient pas identifier les vendeurs d'une période à l'autre. Les unités d'une période à l'autre sont des unités distinctes.

Afin de tester les hypothèses quant à la demande d'information sur la qualité des produits, on crée les variables suivantes:

- prix_seuil15 est égale au prix si le prix est inférieur à 140 ou supérieur à 320 et 0 sinon

- prix_seuil2 est égale au prix si le prix est compris entre 140 et 170 et 0 sinon

- prix_seuil3 est égale au prix si le prix est compris entre 170 et 260 et 0 sinon

- prix_seuil4 est égale au prix si le prix est compris entre 260 et 320 et 0 sinon

L'estimation est présentée dans le tableau 8.

\section{[Insérer le tableau 8]}

La probabilité de demander de l'information sur une unité est significativement inférieure (1\%) dans le traitement High par rapport au traitement Low: cela confirme la loi de la demande d'information. Plus le prix de l'information est élevé, plus la demande est faible.

Concernant l'influence du prix des unités, les résultats vont dans le sens des prédictions. Ainsi, le prix des unités n'a pas d'influence sur la demande d'information sur la qualité des unités lorsque le prix est inférieur à 140 ou supérieur à 320. Par ailleurs, la demande d'information est positivement et significativement (1\%) influencée par le prix entre 140 et 320. Cette influence positive est la plus forte pour un prix compris entre 170 et 260 et la plus faible pour un prix compris entre 140 et 170. 


\section{Conclusion}

L'objectif de cet article était la mise en évidence de l'inefficacité des marchés en présence de coûts d'information élevés sur la qualité des biens. La partie empirique révèle une inefficacité forte des marchés lorsque le coût d'information est élevé. Pour les acheteurs, même un coût d'information faible sur la qualité réduit considérablement les surplus. La probabilité de proposer de la qualité supérieure est d'autant plus forte que l'espérance de gain est élevée. Comme l'efficacité, elle décroît fortement avec les coûts d'information. Enfin, la probabilité de demander l'information sur la qualité est d'autant plus forte que l'information est coûteuse. Et elle dépend du prix des unités dans une relation non linéaire. Les résultats montrent bien les risques présentés par les écolabels si ceux-ci présentent des coûts d"information élevés.

L'expérience réalisée ici est préliminaire. Elle visait à mettre en évidence l'inefficacité du marché lorsque les coûts d'acquisition de l'information sont exclusivement supportés par les acheteurs. Elle constitue une première étape vers l'étude de mécanismes permettant d'améliorer l'efficacité de la transaction en atténuant la dissipation du consentement à payer des consommateurs (réputation, auto-déclarations, certification par tierce partie). L'analyse peut être généralisée à l'ensemble des caractéristiques de croyance tels que la présence d'OGM, la qualité sanitaire ou le bien être animal. Une extension possible peut être l'intégration d'une probabilité d'erreur dans l'information achetée, puisque dans la réalité, les consommateurs, à travers la lecture des étiquettes, des revues de consommateurs, etc., acquièrent rarement une information certaine.

Nous suggérons des pistes d'améliorations de nos travaux. D'une part, étant donné le retour imparfait sur les gains qu'avaient les acheteurs (à moins d'acheter l'information à chaque période), il aurait été intéressant de mesurer les croyances des acheteurs par rapport à la qualité des produits étant donné leur prix. Des expériences incluant ce type de mesure ont déjà été réalisées (Charness et Dufwenberg, 2004). D'autre part, notre protocole était un environnement simplifié. On peut noter que dans la réalité les coûts d'information sont hétérogènes au sein des acheteurs. Ils dépendent de l'accessibilité de l'information ainsi que des capacités individuelles de traitement de l'information. Une autre source d'hétérogénéité qui n'est pas considérée ici réside dans les goûts des acheteurs, leurs préférences pour la qualité environnementale. Cette expérience ouvre des voies de recherche prometteuses. 


\section{Bibliographie}

Akerlof G., 1970, The Market for 'Lemons': Quality Uncertainty and the Market Mechanisms, Quart. J. Econ., 84:488-500.

Andersen E. S., Philipsen K., "The Evolution of Credence Goods in Customer Markets: Exchanging 'Pigs in Pokes'", DRUID Winter Seminar, Middelfart, 8-10 January 1998, 19p.

Bester H., Ritzberger K., 2001, Strategic Pricing, Signalling, and Information Acquisition, Int. J. Ind. Organ., 19:1347-1473.

Brannon J.I., Gorman M.F., 2002, The Effects of Information Costs on Search and Convergence in Experimental markets, J. Econ. Behav. Organ., 47:375-390

Cason T., Gangadharan L., 2002, Environmental Labeling and Incomplete Consumer Information in Laboratory Markets, J. Environ. Econ. Manage., 43(1):113-34.

Caswell J., Mojduzska E., 1996, Using Informational Labeling to Influence the Market for Quality in Food Products, Amer. J. Agr. Econ., 78:1248-53.

Charness G., Dufwenberg M., 2004, Promises and Partnership, UCLA Department of Economics, Levine's Bibliography.

Cho B.-H., Hooker N.H., 2002, A Note on Three Qualities: Search, Experience and Credence Attributes. Working paper AEDE-WP-0027-02, 20 p.

Darby M., Karni E., 1973, Free Competition and the Optimal Amount of Fraud, J. Law Econ., 16:67-88.

Davenport T.H., Beck J.C., 2001, The Attention Economy, Harvard Business School Press, MA, USA.

DeJong D., Forsythe R., Lundholm R., Uecker W., 1985, A Laboratory Investigation of the Moral Hazard Problem in an Agency Relationship, J. Acc. Res., 23:81-120.

Eggertson T., 1990, Economic Behavior and Institutions. Cambridge: Cambridge University Press.

Ford G.T., Smith D.B., Swasy J.L., 1988, An Empirical Test of the Search, Experience and Credence Attributes Framework, Advances in Consumer Research, 15 : 239-48.

Grossman S. J., Stiglitz J. E., 1980, On the Impossibility of Informationally Efficient Markets, Amer. Econ. Rev., 70(3):393-408. 
Holt C., 1995, Industrial Organization :A Survey of Laboratory Research, In : Handbook of Experimental Economics, Kagel J., Roth A.., eds, Princeton University Press, Princeton, NJ, USA, 721p, pp.349-443.

Karl H., Orwat C., 1999. Economic aspects of environmental labelling. In The International Yearbook of Environmental and Resource Economics 1999-2000. Folmer H, Tietenberg T (eds). Edward Elgar: Adelshot.

Keser C., Willinger M., 2002, Experiments on Moral Hazard and Incentives: Reciprocity and Surplus Sharing, In : The Economics of Contracts, Brousseau E., Glachant J.-M., eds, Cambridge University Press, 2002.

Keser C., Willinger M., 2000, La théorie des contrats dans un contexte expérimental : un survol des expériences sur les relations "principal-agent", Revue Econ. Ind., 92:237-53.

Kihlstrom R.E., 1974, A General Theory of Demand for Information about Product Quality, J. Econ. Theory, 8(4):413-39.

Krouse C., 1990, Theory of Industrial Economics, Oxford: Basil Blackwell, 1990.

Loader R., Hobbs J.E., 1999, Strategic Responses to Food Safety Legislation, Food Pol., 24:685-706.

Lynch M., Miller R., Plott C., Porter R., 1986, Product Quality, Consumer Information, and 'Lemons' in Experimental Markets, In P. M. Ippolito and D. T. Scheffman, Empirical Approaches to Consumer Protection Economics, United States Federal Trade Commission, Bureau of Economics, 251-306.

Lynch M., Miller R., Plott C., Porter R., 1991, Product Quality, Information Efficiency, and Regulations in Experimental Markets, Research in Experimental Economics, 4:269-318.

McCluskey J., 2000, A Game Theoretic Approach to Organic Foods: An Analysis of Asymmetric Information and Policy, Agr. Resource Econ. Rev., 29(1):1-9.

Miller R., Plott C., 1985, Product Quality Signaling in Experimental Markets, Econometrica, 53(4):837-72.

Nadaï A., 1998, Concurrence dans la qualification environnementale des produits, Revue Econ. Ind., 83:197-212.

Nelson P., 1970, Information and Consumer Behavior, J. Polit. Economy, 78:311-29.

Noussair C., Plott C., Riezman R., 1995, An Experimental Investigation of the Patterns of International Trade, Amer. Econ. Rev., 85(3):462-91. 
Plott C., Wilde L., 1982, Professional Diagnosis vs. Self-Diagnosis: an Experimental Examination of Some Special Features of Markets with Uncertainty, Research in Experimental Economics, 2:63-112.

Stigler G. J., 1961, The Economics of Information, J. Polit. Economy, 69(3):213-25.

Teisl M.F., Roe B., Hicks R.L., 2002, Can Eco-labels Tune a Market? Evidence from Dolphin-Safe Labeling, J. Environ. Econ. Manage., 43:339-59. 
Figure 1 : Offre et demande pour les unités $\mathrm{S}$ et I

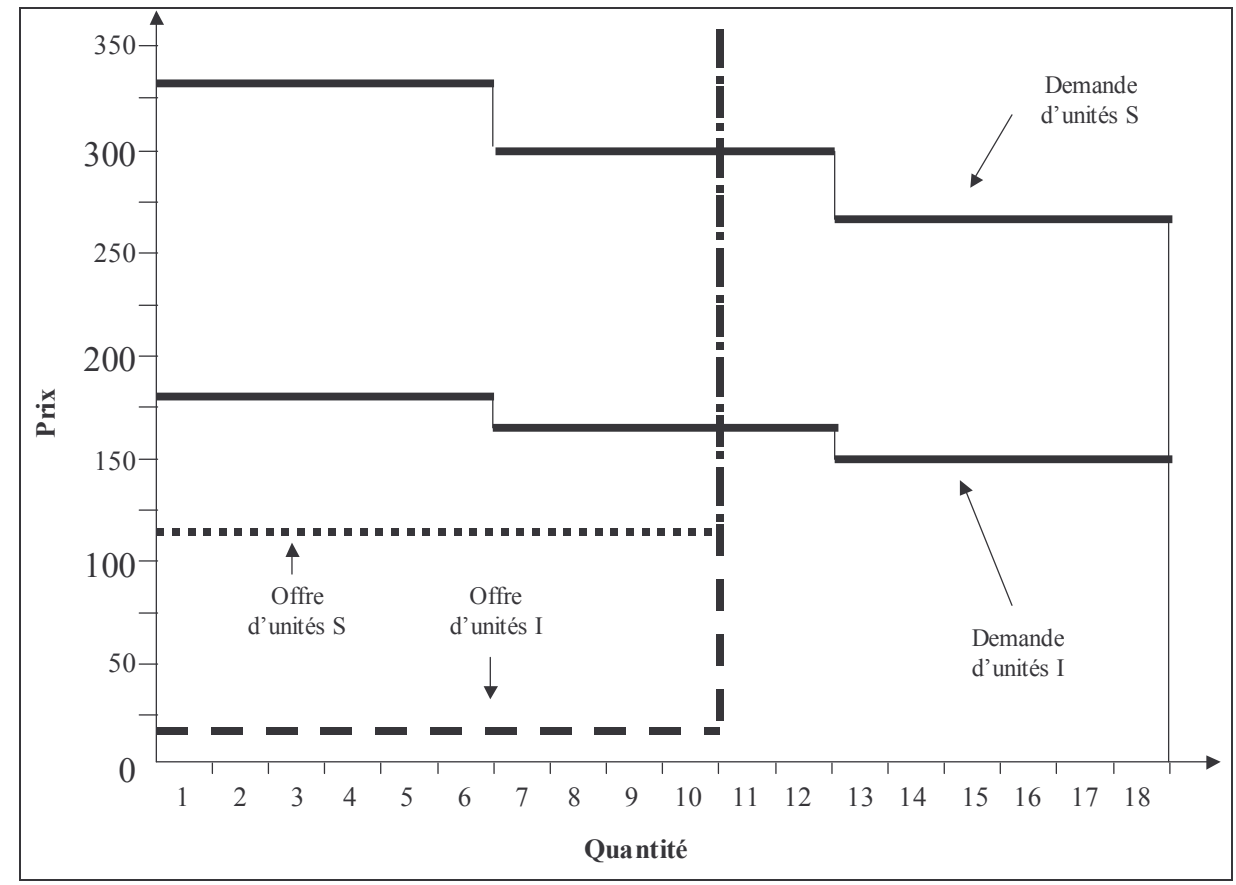

\begin{tabular}{|c|c|c|c|c|c|}
\hline Traitement & Sessions & Lieu & Caractéristiques & $\begin{array}{l}\text { Coût de } \\
\text { l'information } \\
\text { (absolu) }\end{array}$ & $\begin{array}{c}\text { Coût de l'information } \\
\text { (en proportion du consentement à } \\
\text { payer maximal pour une unité } S \\
\text { par rapport à une unité I) }\end{array}$ \\
\hline \multirow[t]{3}{*}{$\operatorname{Ref}$} & A & Dijon & \multirow{3}{*}{$\begin{array}{l}\text { Asymétrie d'information entre } \\
\text { acheteurs et vendeurs }\end{array}$} & \multirow[t]{3}{*}{ 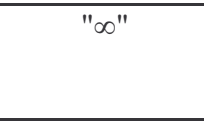 } & \multirow[t]{3}{*}{-} \\
\hline & B & Dijon & & & \\
\hline & $\mathrm{C}$ & Rennes & & & \\
\hline \multirow[t]{3}{*}{ High } & $\mathrm{D}$ & Dijon & \multirow{3}{*}{$\begin{array}{l}\text { Possibilité pour les acheteurs } \\
\text { d'obtenir de l'information à un } \\
\text { coût élevé }\end{array}$} & \multirow[t]{3}{*}{100 points } & \multirow[t]{3}{*}{ Environ $66 \%$} \\
\hline & E & Dijon & & & \\
\hline & $\mathrm{F}$ & Dijon & & & \\
\hline \multirow[t]{3}{*}{ Low } & $\mathrm{G}$ & Dijon & \multirow{3}{*}{$\begin{array}{l}\text { Possibilité pour les acheteurs } \\
\text { d'obtenir de l'information à un } \\
\text { faible coût }\end{array}$} & \multirow[t]{3}{*}{15 points } & \multirow[t]{3}{*}{$10 \%$} \\
\hline & $\mathrm{H}$ & Dijon & & & \\
\hline & I & Dijon & & & \\
\hline
\end{tabular}

Tableau 1: Traitements

\begin{tabular}{|c|c|c|c|c|c|c|c|c|c|c|c|c|}
\hline \multirow[b]{3}{*}{ Périodes } & \multicolumn{8}{|c|}{ Nombre moyen d'unités par période } & \multicolumn{4}{|c|}{ Efficacité moyenne } \\
\hline & \multicolumn{2}{|c|}{$\operatorname{Ref}^{\mathrm{a}}$} & \multicolumn{2}{|c|}{ High } & \multicolumn{2}{|c|}{ High $^{\mathrm{b}}$} & \multicolumn{2}{|c|}{ Low } & \multirow{2}{*}{ Ref } & \multirow{2}{*}{ High } & \multirow{2}{*}{ High $^{b}$} & \multirow{2}{*}{ Low } \\
\hline & I & $\mathrm{S}$ & $\mathrm{I}$ & $\mathrm{S}$ & I & $\mathrm{S}$ & $\mathrm{I}$ & $\mathrm{S}$ & & & & \\
\hline $5-32$ & 6,4 & 0,2 & 7 & 1,4 & 7,8 & 0,7 & 6,5 & 1,3 & 0,729 & 0,893 & 0,876 & 0,869 \\
\hline $23-32$ & 7,9 & 0 & 7,1 & 1,6 & 8,2 & 0,7 & 7,1 & 1,5 & 0,602 & 0,655 & 0,634 & 0,687 \\
\hline $28-32$ & 8 & 0 & 7,7 & 1,3 & 9,2 & 0,4 & 7,1 & 1,6 & 0,615 & 0,691 & 0,692 & 0,699 \\
\hline
\end{tabular}

Tableau 2: Nombre moyen d'unités $S$ et I échangées par période et efficacité moyenne du marché et par traitement 


\begin{tabular}{|l|c|l|}
\hline Variable & Signification & Description \\
\hline High & - & Indicatrice pour le traitement High \\
\hline Low & - & Indicatrice pour le traitement Low \\
\hline Ref_init & $\frac{1}{t}$ & $\begin{array}{l}\text { Variable qui capte l'effet "périodes initiales" du } \\
\text { traitement de référence par rapport à l'effet "périodes } \\
\text { finales" du traitement Ref }\end{array}$ \\
\hline High_init & $H i g h \times \frac{1}{t}$ & $\begin{array}{l}\text { Variable qui capte l'effet "périodes initiales" du } \\
\text { traitement High par rapport au traitement Ref }\end{array}$ \\
\hline High_fin & $I I I \times \frac{(t-1)}{t}$ & $\begin{array}{l}\text { Variable qui capte l'effet "périodes finales" du } \\
\text { traitement High par rapport au traitement Ref }\end{array}$ \\
\hline Low_init & $I I \times \frac{1}{t}$ & $\begin{array}{l}\text { Variable qui capte l'effet "périodes initiales" du } \\
\text { traitement Low par rapport au traitement Ref }\end{array}$ \\
\hline Low_fin & $I I \times \frac{(t-1)}{t}$ & $\begin{array}{l}\text { Variable qui capte l'effet "périodes finales" du } \\
\text { traitement Low par rapport au traitement Ref }\end{array}$ \\
\hline RATIO & $\frac{E\left(\pi_{S}^{N}\right)}{E\left(\pi_{I}^{N}\right)}$ & $\begin{array}{l}\text { Rapport des profits espérés de vendre des unités S } \\
\text { plutôt que des unités I }\end{array}$ \\
\hline
\end{tabular}

Tableau 3 : Variables indépendantes utilisées dans les différents modèles

\begin{tabular}{|l|l|l|l|}
\hline Périodes & \multicolumn{3}{|c|}{$\mathbf{2 1 6}$} \\
\hline Observations & \multicolumn{3}{|c|}{$\mathbf{2 1 6}$} \\
\hline $\begin{array}{l}\text { Variables } \\
\text { indépendantes }\end{array}$ & $\begin{array}{l}\text { Paramètre } \\
\text { estimé }\end{array}$ & $\begin{array}{l}\text { Ecart- } \\
\text { type }\end{array}$ & $\begin{array}{l}\text { Test } \\
\text { p-value }\end{array}$ \\
\hline Constante & $0,5738 * * *$ & 0,03301 & $<, 0001$ \\
\hline Ref_init & $-0,9618 * *$ & 0,3840 & 0,0130 \\
\hline High_init & 0,7095 & 0,5517 & 0,1999 \\
\hline High_fin & 0,07506 & 0,04987 & 0,1338 \\
\hline Low_init & $-0,9931 * *$ & 0,4951 & 0,0462 \\
\hline Low_fin & $0,1986 * * *$ & 0,04495 & $<, 0001$ \\
\hline -2Log L & \multicolumn{3}{|c|}{$-221,2$} \\
\hline AIC & \multicolumn{3}{|c|}{$-207,2$} \\
\hline BIC & $* 6,6$ \\
\hline
\end{tabular}

Tableau 4 : Efficacité du marché (effet aléatoire session) 


\begin{tabular}{|l|l|l|r|}
\hline Périodes & \multicolumn{3}{|c|}{$\mathbf{5 - 3 2}$} \\
\hline Observations & \multicolumn{3}{|c|}{$\mathbf{2 1 6}$} \\
\hline $\begin{array}{l}\text { Variables } \\
\text { indépendantes }\end{array}$ & $\begin{array}{l}\text { Paramètre } \\
\text { estimé }\end{array}$ & $\begin{array}{l}\text { Ecart- } \\
\text { type }\end{array}$ & $\begin{array}{l}\text { Test } \\
\text { p-value }\end{array}$ \\
\hline Constante & $0,5485 * * *$ & 0,04631 & $<, 0001$ \\
\hline Ref_init & $1,7037 * * *$ & 0,4293 & $<, 0001$ \\
\hline High_init & $-2,1288 * * *$ & 0,6157 & 0,0007 \\
\hline High_fin & $0,1579 * *$ & 0,07081 & 0,0268 \\
\hline Low_init & $-3,3664 * * *$ & 0,5529 & $<, 0001$ \\
\hline Low_fin & $0,2061 * * *$ & 0,06370 & 0,0014 \\
\hline -2Log L & \multicolumn{3}{|c|}{$-166,2$} \\
\hline AIC & \multicolumn{3}{|c|}{$-150,2$} \\
\hline BIC & \multicolumn{3}{|c|}{$-149,6$} \\
\hline
\end{tabular}

Tableau 5 : Efficacité du marché pour les vendeurs (effet aléatoire session)

\begin{tabular}{|l|c|r|r|}
\hline Périodes & \multicolumn{3}{|c|}{$\mathbf{5 - 3 2}$} \\
\hline Observations & \multicolumn{3}{|c|}{$\mathbf{2 1 6}$} \\
\hline $\begin{array}{l}\text { Variables } \\
\text { indépendantes }\end{array}$ & $\begin{array}{l}\text { Paramètre } \\
\text { estimé }\end{array}$ & $\begin{array}{l}\text { Ecart- } \\
\text { type }\end{array}$ & $\begin{array}{l}\text { Test } \\
\text { p-value }\end{array}$ \\
\hline Constante & $0,7388^{*}$ & 0,3281 & 0,0741 \\
\hline Ref_init & $-26,8968^{* * *}$ & 3,1836 & $<, 0001$ \\
\hline High_init & $28,4582^{* * *}$ & 4,5651 & $<, 0001$ \\
\hline High_fin & $-0,6651$ & 0,5002 & 0,1851 \\
\hline Low_init & $22,0965^{* * *}$ & 4,0991 & $<, 0001$ \\
\hline Low_fin & 0,2118 & 0,4502 & 0,6385 \\
\hline -2Log L & \multicolumn{3}{|c|}{698,2} \\
\hline AIC & \multicolumn{3}{|c|}{714,2} \\
\hline BIC & 714,8 \\
\hline$*:$ significatif à $10 \% * *$ à $5 \% * *: 1 \%$
\end{tabular}

Tableau 6 : Efficacité du marché pour les acheteurs (effet aléatoire session)

\begin{tabular}{|l|c|c|c|}
\hline Périodes & \multicolumn{3}{|c|}{$\mathbf{5 - 3 2}$} \\
\hline Observations & \multicolumn{3}{|c|}{$\mathbf{1 0 8 0}$} \\
\hline $\begin{array}{l}\text { Variables } \\
\text { indépendantes }\end{array}$ & $\begin{array}{l}\text { Paramètre } \\
\text { estimé }\end{array}$ & $\begin{array}{l}\text { Ecart- } \\
\text { type }\end{array}$ & $\begin{array}{l}\text { Test } \\
\text { p-value }\end{array}$ \\
\hline Constante & $-4,5180^{* * *}$ & 0,6048 & $<, 0001$ \\
\hline Ref_init & $16,8398^{* * *}$ & 3,5888 & $<, 0001$ \\
\hline High_init & $-7,0979$ & 5,3772 & 0,1945 \\
\hline High_fin & 0,4455 & 0,8456 & 0,6013 \\
\hline Low_init & $-10,7831^{* *}$ & 4,4928 & 0,0213 \\
\hline Low_fin & $1,4146^{*}$ & 0,7103 & 0,0534 \\
\hline RATIO & $0,7557^{* *}$ & 0,3367 & 0,0305 \\
\hline -2Log L & \multicolumn{3}{|c|}{704} \\
\hline AIC & \multicolumn{3}{|c|}{730} \\
\hline BIC & \multicolumn{3}{|c|}{} \\
\hline
\end{tabular}

Tableau 7 : Probabilité de proposer des unités de type $\mathrm{S}$ pour les vendeurs 


\begin{tabular}{|c|c|c|c|}
\hline Périodes & \multicolumn{3}{|c|}{ 5-32 } \\
\hline Observations & \multicolumn{3}{|c|}{700} \\
\hline Observations où $\mathrm{DEM}=1$ & \multicolumn{3}{|c|}{208} \\
\hline Variables indépendantes & $\begin{array}{l}\text { Paramètre } \\
\text { estimé }\end{array}$ & $\begin{array}{l}\text { Ecart- } \\
\text { type }\end{array}$ & Test $\chi^{2}$ \\
\hline Constante & $-4,8221 * * *$ & 1,0905 & $<, 0001$ \\
\hline High & $-2,6264 * * *$ & 0,3130 & $<, 0001$ \\
\hline prix_seuil15 & 0,00162 & 0,00469 & 0,7299 \\
\hline prix_seuil2 & $0,0182 * * *$ & 0,00704 & 0,0098 \\
\hline prix_seuil3 & $0,0302 * * *$ & 0,00550 & $<, 0001$ \\
\hline prix_seuil4 & $0,0255 * * *$ & 0,00450 & $<, 0001$ \\
\hline $\mathbf{T}$ & 0,00211 & 0,0159 & 0,8943 \\
\hline$-2 \log \mathrm{L}$ & \multicolumn{3}{|c|}{462,6} \\
\hline $\begin{array}{l}\text {-2Log } L \text { avec constante } \\
\text { uniquement }\end{array}$ & \multicolumn{3}{|c|}{851,8} \\
\hline $\begin{array}{l}\text { Rapport de vraisemblance } \\
\text { et test }\end{array}$ & \multicolumn{3}{|c|}{$389,2 * * *$} \\
\hline $\begin{array}{l}\text { Pourcentage de cas } \\
\text { correctement prédits }\end{array}$ & \multicolumn{3}{|c|}{$90,5 \%$} \\
\hline
\end{tabular}

Tableau 8 : Probabilité de demander de l'information sur une unité

Encadré 1 : Prise en compte du phénomène de convergence des marchés dans l'analyse économétrique (Noussair et al., 1995)

Si on définit $y_{i t}$ comme la variable dépendante mesurée sous plusieurs traitements $i=1, \ldots, n$ et fonction du temps, la spécification du modèle est la suivante :

$y_{i t}=B_{11} D_{1}\left(\frac{1}{t}\right)+B_{12} D_{2}\left(\frac{1}{t}\right)+\ldots+B_{1 i} D_{i}\left(\frac{1}{t}\right)+\ldots+B_{1 n} D_{n}\left(\frac{1}{t}\right)+B_{2} \frac{(t-1)}{t}+u$

avec $t$, le temps mesuré comme le nombre de périodes dans chaque traitement; $D_{i}$, une indicatrice qui prend la valeur 1 pour le traitement i et la valeur 0 sinon; $B_{1 t}$, la mesure de l'existence éventuelle d'un phénomène de convergence.

Si $t=1$, alors la valeur de la variable dépendante est $B_{1 i}$ pour l'expérience $i . B_{2}$ est l'asymptote de la variable dépendante. Lorsque $t$ augmente, le poids de $B_{1 i}$ devient faible car $1 / t$ tend alors vers 0 , tandis que le poids de $B_{2}$ augmente car $(t-1) / t$ tend vers $1 . u$ est le terme d'erreur aléatoire. 


\section{Annexe: exemple d'instructions INSTRUCTIONS EXPERIENCE DE MARCHE (Coût de l'information 100 points)}

\section{Introduction}

Vous allez participer à une expérience concernant les décisions individuelles. Les instructions sont simples et si vous les suivez avec attention et faites de bons choix, vous gagnerez de l'argent qui vous sera versé en espèces à la fin de l'expérience.

\section{VOUS NE DEVEZ PAS COMMUNIQUER ENTRE VOUS}

Vous allez participez à un marché dans lequel vous serez un participant dans une série de $\mathbf{3 2}$ périodes d'échange. On vous distribuera 2 feuilles intitulées "Feuille d'Informations" et "Feuille d'Enregistrement". Vous ne devez révéler ces informations à personne. C'est votre information privée. Tous les échanges et tous les gains dans cette expérience seront calculés en points ou en UME (Unités de Monnaie Expérimentale). Votre Feuille d'Information indiquera combien d'euros vaut un point et combien de points vaut un euro. Ne révélez ces nombres à personne. A la fin de l'expérience, vos points seront convertis en euros, et vous serez payés en euros. Remarquez que plus vous gagnez de points, plus vous gagnez d'euros.

Au cours de chaque période d'échange, vous serez libres de vendre ou d'acheter des unités S. Au début de l'expérience, vous apprendrez si vous êtes un vendeur ou un acheteur. Vous garderez ce rôle tout au long de l'expérience. Dans cette expérience, il y a 5 vendeurs et 6 acheteurs.

\section{Acheteurs}

A chaque période d'échange, les acheteurs sont libres d'acheter auprès de n'importe quel(s) vendeur(s) jusqu'à 3 unités maximum.

Le nombre de points que rapporte chaque unité achetée est appelé « valeur de reprise ». Elle dépend du type de l'unité. Il y a 2 types d'unités ( $\mathbf{I}=$ Inférieur et $\mathbf{S}=$ Supérieur) et la valeur pour l'acheteur d'une unité $\mathbf{S}$ est supérieure à la valeur d'une unité I.

Au moment où les acheteurs achètent une unité, ils n'en connaissent pas le type mais uniquement le prix. A chaque période d'échange, avant l'achat, les acheteurs ont la possibilité d'obtenir de l'information sur le type des unités $\mathrm{S}$ qui sont offertes par chacun des vendeurs. Cette information a un coût de $\mathbf{1 0 0}$ points.

La session se compose de 4 parties :

Partie 1 : les périodes 1 à 4

Les acheteurs connaîtront le type des unités $\mathrm{S}$ achetées à la fin de chaque période d'échange donc après chaque achat.

Partie 2 : les périodes 5 à 8

Les acheteurs ne connaîtront le type des unités $\mathrm{S}$ achetées dans chacune des périodes et donc leurs gains qu'après ces 4 périodes.

Partie 3 : les périodes 9 à 16

Les acheteurs ne connaîtront le type des unités $\mathrm{S}$ achetées dans chacune des périodes et donc leurs gains qu'après ces 8 périodes.

Partie 4 : les périodes 17 à 32

Les acheteurs ne connaîtront le type des unités $\mathrm{S}$ achetées dans chacune des périodes et donc leurs gains qu'après ces 16 périodes.

Les acheteurs indiqueront soigneusement et suivront, période après période, les décisions qu'ils ont prises sur leur « Feuille d'Enregistrement ».

Les acheteurs ont aussi la possibilité de n'acheter aucune unité dans une période d'échange. Ils recevront alors un gain alternatif de non achat comme indiqué sur leur Feuille d'Enregistrement.

La valeur de reprise des unités $\mathrm{S}$ achetées est la valeur que vous pourrez retirer de ceunités S. Votre Feuille d'Information personnelle inclut 2 valeurs de reprise pour les types I ou S. Ces valeurs décroissent avec le nombre d'unités achetées par période. Si vous avez déjà acheté une unité de type $\mathrm{S}$ et que dans la même période, vous achetez une $2^{\text {ème }}$ unité de type $S$, cette $2^{\text {ème }}$ unité aura une valeur de reprise moindre.

Par exemple, supposons que vos valeurs de reprise soient :

\begin{tabular}{|l|l|l|}
\hline & Type S & Type I \\
\hline $\mathbf{1}^{\text {ère }}$ unité DE CE TYPE achetée dans la période & 4000 & 1500 \\
\hline $\mathbf{2}^{\text {ème }}$ unité DE CE TYPE achetée dans la période & 3000 & 1000 \\
\hline $\mathbf{3}^{\text {ème }}$ unité DE CE TYPE achetée dans la période & 2000 & 500 \\
\hline
\end{tabular}

Si les 3 unités achetées dans la période sont comme suit :

la $1^{\text {ère }}$ unité ACHETEE DANS LA PÉRIODE est de type I,

la $2^{\text {ème }}$ unité ACHETEE DANS LA PÉRIODE est de type $\mathrm{S}$ et 
la $3^{\text {ème }}$ unité ACHETEE DANS LA PÉRIODE est de type I....

alors, la $1^{\text {ère }}$ unité achetée rapportera 1500 (c'est la $1^{\text {ère }}$ unité I achetée), la $2^{\text {ème }}$ unité achetée rapportera 4000 (c'est la $1^{\text {ère }}$ unité $\mathrm{S}$ achetée) et la $3^{\text {ème }}$ unité achetée rapportera 1000 (c'est la $2^{\text {ème }}$ unité I achetée).

La valeur de reprise totale sera alors : $1500+4000+1000=6500$ points pour cette période.

Vos gains sont calculés en faisant la différence entre d'une part, la ou les valeurs de reprise et d'autre part, le prix payé pour l'unité et le coût d'information.

[Vos gains $=($ valeur(s) de reprise des unités $S$ achetées $)-($ prix d'achat $)-($ coût d'information $)]$

Supposons que vous achetiez une unité de type I au prix de 1000 et que vous achetiez aussi une unité de type S au prix de 1200. Vous avez également acheté de l'information sur l'unité $\mathrm{S}$ à 400 points. Vos gains sont alors :

\begin{tabular}{|lll|}
\hline Pour l'unité de type I : & $1500-1000$ & $=500$ \\
Pour l'unité de type S : & $4000-1200-400$ & $=\underline{2400}$ \\
& TOTAL: & $\mathbf{2 9 0 0}$ \\
\hline
\end{tabular}

En plus de ces gains, vous recevrez automatiquement sans conditions un gain additionnel à chaque période et un gain initial en début d'expérience. Ces chiffres sont indiqués sur votre Feuille d'Enregistrement.

A chaque période et pour chaque unité achetée, les acheteurs remplissent soigneusement leur Feuille d'Enregistrement indiquant le numéro du vendeur, le prix d'achat ainsi que le type connu après chaque période (partie 1), après 4 périodes (partie 2), après 8 périodes d'échange (partie 3 ) ou après 16 périodes d'échange (partie 4).

L'expérimentateur vous aidera à remplir votre Feuille d'Enregistrement pendant les premières périodes.

\section{Vendeurs}

A chaque période d'échange, les vendeurs peuvent vendre à n'importe quel acheteur un maximum de $\mathbf{2}$ unités du même type I ou S.

Votre Feuille d'Information indiquera le coût de production d'une unité de type I et le coût de production d'une unité de type $S$.

Le coût de production d'une unité de type $S$ est supérieur au coût de production d'une unité de type $I$.

Si vous ne vendez aucune unité à une période d'échange donnée, vous ne subissez pas le coût de production des unités $\mathbf{S}$ non vendues. Vos profits sont simplement zéro.

Les profits ou pertes à chaque achat sont calculés en faisant la différence entre le prix auquel vous avez vendu votre unité et son coût de production :

[vos gains $=($ prix de vente de l'unité $)-($ coût de production de l'unité $)]$

Supposez par exemple que le coût de production d'une unité de type S soit 1000 et que vous proposiez à la vente 2 unités de type $\mathrm{S}$ au prix unitaire de 1600. On vous achète une unité, vos gains sont alors :

$\begin{array}{lll}\text { Pour l'unité vendue : } & 1600-1000= & 600\end{array}$

Pour l'unité non vendue : $\quad 0 \quad \frac{0}{600}$

Les profits totaux par période d'échange sont calculés en ajoutant les profits ou pertes dues aux ventes réalisées à chaque période. Les vendeurs pourront suivre à l'écran leurs profits par période et leur profit cumulé. Ils rempliront période après période leur Feuille d'Enregistrement.

L'expérimentateur vous aidera à remplir votre Feuille d'Enregistrement pendant les premières périodes.

\section{Procédure d'échange}

Au début de chaque période, les vendeurs font une offre pour la période en cours : ils proposent 1 ou 2 unités dont ils choisissent le type (I ou S) et le prix.

Le tableau des différentes offres (prix et nombre d'unités) est transmis aux acheteurs. LES ACHETEURS SONT ENSUITE SELECTIONNES DE MANIERE ALEATOIRE POUR REALISER LEURS ACHATS.

Chaque acheteur choisit d'acheter ou non de l'information. Ensuite, chaque acheteur accepte ou non d'acheter des unités S (jusqu'à 3). Il remplit soigneusement sa Feuille d'Enregistrement.

Lorsque tous les acheteurs ont eu la possibilité d'acheter ou que toutes les unités S sont vendues, une nouvelle période d'échange a lieu. Pendant la partie 1, les types des unités $\mathrm{S}$ sont révélés après chaque période. Dans la 
partie 2, les types des unités $\mathrm{S}$ achetées ne sont révélées aux acheteurs qu'après 4 périodes. Dans la partie 3 , les types des unités $\mathrm{S}$ achetées ne sont révélées aux acheteurs qu'après 8 périodes. Dans la partie 4 , les types des unités $\mathrm{S}$ achetées ne sont révélées aux acheteurs qu'après 16 périodes.

\section{Résumé}

- Gains des acheteurs = Valeur de reprise de l'unité - Prix d'achat de l'unité - coût de l'information.

- Gain des vendeurs par unité = Prix de vente de l'unité - Coût de production de l'unité

- Les vendeurs font une offre à chaque période, en choisissant un prix, une quantité et un type (I ou S).

- Les acheteurs reçoivent le tableau des offres (prix et nombre d'unités proposées) et choisissent ou non d'acheter de l'information et d'acheter ou non des unités $\mathrm{S}$ aux prix offerts.

- Les acheteurs peuvent choisir de ne pas acheter et recevoir un gain de « non achat » pour cette période.

Y a-t-il des questions? 